the gut where the toxicity is most marked. If we acidify and boil the contents of the lower end of the ileum, filter, and add alcohol or tannic acid to the filtrate, it gives no appreciable precipitate.

'The results of perfusion of the toad's heart give us a much more reliable indication than those of intravenous injection in the mammal. In the latter the quantity injected is small and the time of action short. It is reasonable to assume that prolonged absorption even in minute quantities would produce effects on the mammalian heart similar to those immediately produced in the toad's heart by comparatively strong concentration. The excised mammalian heart may prove suitable for perfusion with more concentrated solutions, but the difficulties of technique would not be light.

Many conditions of irregularity and block observed in the human heart in Stokes-Adams and other affections seem to fall into line with the action of these substances. Osler? says that poisons arising in the intestine may cause arrhythmia. Flatulence, he says, may cause it reflexly. A neurotic group of cases is described where no post-mortem lesions are found. In the matter of treatment the same author makes brief reference to dieting and the importance of avoiding full meals and flatulency.

A systematic course of treatment of the alimentary canal antiseptically and otherwise should be of the greatest service in some of these cases. Theoretically there are substantial grounds for the adoption of such a course, the arranging of the practical details of which is, however, in the province of the observant practitioner.

Cork.

\section{KALA-AZAR IN SOLDIERS RETURNING FROM MALTA.}

BY GORDON R. WARD, M:D. LOND., CAPTAIN, R.A.M.C. (S.R.).

THIS preliminary note is based on two cases which have come under my notice during the last 12 months, and is written with the hope of drawing early attention to the following facts :-

1. That cases of kala-azar are to be expected among men returning from Malta and who have never been farther East than that point.

2. That the diagnosis is likely to be very difficult to those unacquainted with tropical diseases.

3. That the prognosis must not necessarily be regarded as hopeless.

These facts may be well known to those who have had the opportunity of studying tropical medicine, but they deal with a field of study necessarily almost unknown to the average general practitioner, yet it is the general practitioner who is now treating the majority of the returned troops, and there can be little doubt that he will have to practise, after the war, amongst diseases which have hitherto been merely names to him. It is hoped that the writer's experience may be of some assistance to others.

Owing to certain anomalies in the first case and to the fact that the second case is now under treatment no attempt can be made at present to give a full description. The diagnosis and treatment are of first importance, but these must be prefaced by a few more general remarks.

Kala-azar (the " black fever ") is well known in India, and until the causal parasite-the Leishmania donovani-was discovered by the R.A.M.C. officers whose name it bears was commonly supposed to be a manifestation of malaria. This fate, of course, has been that of most varieties of splenomegaly in the tropics and even, at one time, at home. Beyond India it has been recognised particularly in the Mediterranean, but here it was chiefly met with in children, and this peculiarity has led some people to suppose that the Mediterranean form is a different disease. However this may be, the course of the disease when it attacks adults seems to be the same as that of the Indian form.

The first symptom is either referable to general enfeeblement or to the splenomegaly and may be weakness, dyspnœa, indigestion, and dragging pain. Diarrhoea is a common symptom and well-marked pyrexia is characteristic. The wasting increases, the skin becomes pigmented, the splenomegaly assumes greater prominence, and the patient dies from exhaustion or from some intercurrent infective process. The duration of the disease may be anything from a few months to some years.

The diagnostic problem is somewhat as follows: A patient presents himself with diarrhœe, continuing weakness, or some similar sign which would fit many disease processes. If no thorough examination is made and symptomatic treatment adopted there will be at the best brief subjective improvement. Eventually examination must be made and will disclose a large-probably a very large-spleen. This will naturally elicit a question as to whether the patient has been abroad and, this being answered in the affirmative, the diagnosis of some tropical disease is suggested. This is the stage at which my first patient arrived. He was sent for examination by his commanding officer with the remark that " he had not seemed fit for duty for two or three months, but had refused to report sick." He was noted to be very anæmic and was admitted to hospital, where the splenomegaly was quickly discovered. The medical officer in charge, who held the Diploma in Tropical Medicine, brought it to my notice with the remark that it was a case of leukæmia. On examination marked general pigmentation was noted. This is against a aiagnosis of leukæmia, in which general pigmentation is quite uncommon. A blood examination was made forthwith and revealed a very low leucocyte count. This excluded leukæmia and suggested kala-azar. It did not, however, rule out splenic anæmia. A spleen puncture, however, afforded abundant evidence of the nature of the disease, very many typical parasites being seen.

It is obvious that the conveniences of blood examination and spleen puncture may not always be available, and it is therefore pertinent to inquire how far a definite diagnosis can be arrived at without them. Absolute diagnosis is not possible unless the parasite be found, but the following points in a case of massive splenomegaly are most in favour of kala-azar-viz., residence abroad, general pigmentation, absence of enlarged glands, deafness and failing sight (all of which may be found in leukremia), and the temperature. The latter is peculiar in showing two daily rises, and a four-hourly chart is necessary to show this. The first maximum is reached about noon, the second in the evening.

My second case was admitted to hospital after three or four months of increasing debility. He complained mostly of diarrhœa. When only a few days' temperature readings were available they closely resembled a somewhat typical enteric defervescence. The diarrhœea and splenomegaly were held to be consistent with such a diagnosis, although the extent of the latter was felt to be unusual and disquieting. Major A. Elliot, of the 1st London Field Ambulance, has kindly given me details of another case (also contracted in Malta) in which the same diagnosis was arrived at. It is therefore very important to remember that the splenomegaly of enteric is not nearly so marked as in kala-azar and is not solid to the feel. A hard, massive spleen extending to the umbilicus or to the pubis can never be ascribed to typhoid, nor indeed is it likely to be associated with anything like so acute a process as any of the enteric group. In the second case spleen puncture was performed to place the diagnosis beyond doubt, although reasonably definite parasites were found in the circulating blood.

One other point may be mentioned in diagnosis. The idea that kala-azar was malarial, amongst other factors, gave rise to a very firmly rooted conviction that malaria could produce extreme chronic splenomegaly. Whether this is now held to be the case the writer does not know, but it is significant that Castellani and Chalmers, in their "Manual of Tropical Medicine," give " marked enlargement of the spleen" as a diagnostic point in favour of kala-azar as against malaria. No other authority is immediately available for reference.

As regards prognosis, the same writers are distinctly pessimistic, stating that 70 per cent. die with vigorous quinine treatment and 98 per cent. without this. The first. case mentioned recovered after taking from 60 to 70 grains of quinine daily by the mouth for three months. He has been on full duty for five months since. The second case is now taking 60 grains daily (reached by increments of 6 to 9 grains a day) and there is some slight diminution of the splenomegaly, but his general condition is not improved. He has not been long under observation. ${ }^{1}$ Major Elliot informs me that the case he saw did not have any large doses of quinine and died eventually of cancrum oris. In any case, it is 
obvious that quinine ought to be pushed as much as possible. In neither of my cases did large doses give rise to manifestations of quinine poisoning sufficient to annoy the patient, but when this is the case bromides are said to mitigate the symptoms.

More recently Sir Leonard Rogers and others have used intravenous injections of 2 per cent. tartar emetic in saline, and claim to have better results than by the older methods. The details of technique can be found in sundry papers published in the medical journals during the last year or two.

In conclusion, it may be stated that this note will have achieved its object if the general practitioner, to whom it is addressed, will think of the possibility of kala-azar in the presence of splenomegaly in a soldier returned from abroad. There can be no doubt that the greatest difficulty in diagnosis is the realisation of what the disease may possibly be. Once kala-azar is considered as a possibility the diagnosis presents no particular difficulty.

Sevenoaks, Kent.

\section{ABDOMINO-PERINEAL RESECTION OF RECTUM BY COFFEY'S MODIFICA- TION OF THE TWO-STAGE OPERATION.}

By LAURence C. PANTING, M.D., B.OH.OXon., M.R.C.P. LoND., F.R.C.S. ENG.,

SURGEON TO THE ROYAL CORNWALL INFIRMARY, TRURO.

THE radical operation for cancer of the rectum as elaborated by Ernest Miles ${ }^{1}$ must necessarily be a most serious surgical procedure, for even in his very skilled hands it takes from an hour and a half to two hours to perform, and the mortality reaches 22 per cent., other surgeons admitting a much higher figure. Apart from the grave injury necessarily inflicted on the tissues, the mere duration of anæsthesia for such a prolonged period is serious, and this danger is enhanced by the change in position of the patient required for the performance of the perineal portion of the operation. It is not surprising, therefore, that various plans have been suggested for dividing the operation so that it may be performed in two stages, the abdominal portion constituting the first and the perineal the second stage.

W. Mayo ${ }^{2}$ expresses the opinion that by operating in two stages the mortality may be reduced to one-half of that occurring when it is performed in one. He suggests two plans for the division : the first to perform a colostomy without division of the bowel, followed by Kraske's sacral operation or some modification of it. This has the advantage that it can be employed in cases of obstruction and allows of cleansing the lower and diseased segment, but beyond the fact that it permits exploration for metastases it has none of the advantages of the accepted abdomino-perineal operation, but is really a modification of Kraske's operation. The second method, of which several modifications may here be considered, is that usually adopted. It consists of a first stage at which the pelvic colon is divided and the ends closed, the lower end of the upper segment being brought through the abdominal wall as a permanent colostomy, while the lower segment is freed from its attachments and left to be removed by a perineal operation as a second stage.

In the treatment of the separated lower segment with its attached mesentery and glands several plans have been proposed. Mayo, leaving the lateral attachments of the rectum in the pelvis intact in order to preserve the middle hæmorrhoidal arteries, pushes the whole mass into the lower part of the pelvis and closes the peritoneum over it, leaving it thus until it is removed in the second stage (perineal) operation, six or seven days later. In one case of Mayo's and in several elsewhere recorded the blood-supply has proved insufficient, and perforation of the bowel has led to a fatal result. Another method which he has tried is to divide the bowel with the cautery between clamps, the lower clamp being left in the wound surrounded by a thin rubber tube packed with gauze. Alternatively the bowel may be divided between ligatures, ${ }^{3}$ the end inverted with a purse-string suture, and the peritoneum sewn over it

1 E. Miles : British Journal of Surgery, October, 1914 (full description of his operation).

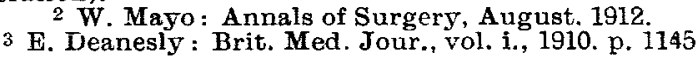

to protect the peritoneal cavity. The danger of sepsis in thus dividing the gut low down in the pelvis in the presence of a sloughing growth is obvious and seems to constitute a very serious disadvantage to the plan. As a further alternative it has been suggested by Coffey ${ }^{4}$ that the lower segment should be inverted through the anus, which prevents any danger of sepsis to the peritoneal cavity since the bowel is not opened. The peritoneal surfaces which are in contact rapidly close the upper end so that when necrosis from loss of blood-supply occurs, there is no longer any avenue open to the pelvic cavity. With these advantages it has, of course, certain limitations; thus, in the case of a large mass of growth it may be technically impossible to perform the inversion and get it through the anus. Another difficulty occurs where the grow th actually involves the region of the anterior reflexion of the peritoneum from the rectum, but this, if sufficiently extended, must render any two-stage operation upon these lines nugatory and that to a greater extent in the other modifications than in this.

As some plan of invagination had for some time seemed to me more hopeful than anything previously described, I at once adopted Coffey's method in the following case which came under my care, and it proved entirely satisfactory.

Mrs. _- aged 49 years, was sent to me in October, 1915 , with a view to operation for cancer of the rectum. She had been suffering for two years from symptoms due chiefly to a polypus which came down every time the bowels acted and bled fairly freely until it was replaced in the rectum. There was also an offensive discharge, but no obstruction and no pain which did not seem easily accounted for by the polypus. She had not appreciably lost flesh, and when seen she was well nourished and not more out of health than might be expected from the constant irritation and discharge. On examination a polypus of about the size of a plum was found attached by a thin pedicle to the anterior wall of the rectum just inside the anus. Some two inches above the anus a soft granular growth was felt, apparently encircling the bowel and reaching upwards as far as the finger could reach. The lower part was but little raised, while the upper part protruded into the lumen of the bowel. Nowhere could any fixation of the rectum be made out; the vagina was normal and the uterus freely movable. The liver was not enlarged, and nowhere could any glandular or metastatic deposit be lelt.

As the case seemed suitable for operation the patient weat to a nursing home, and on the night of her arrival the polypus came down and the opportunity was taken to remove it so that the rectum could be better cleared. The first operation was performed two days later. An incision was made from the umbilicus to the pubes in the middle line, and a preliminary examination made which showed the absence of recognisable metastasis. The pelvic colon was drawn out of the wound and a point selected in its lower part so that as much as possible might remain to form a reservoir for fæces. At this point the bowel was crushed and divided with the cautery between ligatures, the divided end being turned in and closed with a purse-string suture. The mesentery was then divided back to the promontory of the sacrum and the superior hæmorrhoidal artery ligatured. The peritoneum was divided on either side of the rectum down to the base of the broad ligament and the whole mass of rectum with the soft tissues and glands separated from the sacrum as far as the upper end of the coccyx. The anterior reflexion of peritoneum from the rectum was divided, and finally the lateral attachments of the rectum. The portion of gut below the section with its adherent cellular tissue and glands had thus been entirely freed down to a point a little below the anterior reflexion of the peritoneum. A large rubber tube had been prepared, shaped, and stitched so as to form a blunt point at one end and open at the other, and to act as a pilot for this a corresponding glass tube closed and rounded at one end. This tube with its pilot in position was now passed by an assistant through the anus and up the rectum, the end of the tube being guided by the operator's hand in the pelvis. When it was well in the rectum the glass tube was withdrawn and the rubber tube pushed on until it reached the closed end of the bowel. Here it was firmly fixed by stitches passing right through the gut and rubber from side to side. The tube was then slowly withdrawn, invaginating the upper end of the bowel into itself, the walls being meanwhile supported by forceps on each side. This was continued until the whole of the free portion had been invaginated. The cut edges of the peri toneum in the pelvis were drawn together with stitches, and in order to form a second peritoneal layer the uterus was drawn back and stitched to the peritoneum over the sacrum and laterally, the peritoneal floor being thus firmly closed. The lower end of the upper segment was then 